\title{
Algunos alcances sobre el desarrollo y consolidación científica
de la Antropología Psicológica
}

\author{
Carlos A. Haefner
}

\section{INTRODUCCIÓN}

La Antropología Psicológica es la más joven y una de las más pujantes ramas de la Antropología. Sus líneas de estudio han dejado ver una clara coincidencia entre la Antropología y la Psicología, para abordar sistemática y consistentemente el análisis de los fenómenos culturales.

Hasta hace unas décadas la posibilidad de comprometer una orientación psicológica en el trabajo etnográfico presentaba grandes dificultades, especialmente en Europa, donde la tradición durckeniana hacía sentir fuertemente su presencia. Asimismo, el antropólogo interesado en examinar las interrelaciones entre la cultura y la personalidad no contaba con marcos teóricos y herramientas metodológicas apropiadas para este fin.

A comienzos de siglo, en una época en que la antropología estaba más próxima al historicismo y a los problemas de difusionismo cultural, Franz Boas expresaba que el antropólogo debía estudiar la "vida mental" de los pueblos que describía. En forma paulatina muchos de sus discípulos comenzaron a dar forma a lo que hoy conocemos como antropología psicológica, pero debieron ir gradualmente minimizando la natural desconfianza del resto de la comunidad antropológica en lo que al uso de teorías y métodos psicológicos se refiere. Su entusiasmo por generar estudios en esta área los llevó a grandes tentativas de explicación de la formación y características culturales de la personalidad. Sin embargo, la psicologización de la antropología, sólo se produciría a partir del segundo cuarto de siglo. El andar de ésta fue lento y la desconfianza hacia los primeros resultados enormes, debido principalmente a la debilidad, de los procedimientos metodológicos, a lo insustancial de sus contructos teóricos y del recelo al psicologismo. 
Sólo una vez que los investigadores de esta área fueron capaces de depurar y transformar los préstamos metodológicos hechos por la psiquiatría y la psicología clínica, por ejemplo, los test proyectivos, entrevistas en profundidad, biografías, etc., logró, la antropología psicológica transformarse en un sólido campo de estudio.

Actualmente, la antropología psicológica es un prestigioso subcampo de la antropología cultural, que poco tiene que envidiar, en lo que a confiabilidad y representatividad se refiere, a otras especializadas áreas antropológicas. En términos teóricos, abarca temas tan variados que van desde la evolución de la conducta humana y el desenvolvimiento de la "naturaleza humana", hasta la exploración de la percepción y la cognición de pautas interculturales, además de aspectos de cambio cultural. En definitiva, se caracteriza por su interés en distinguir los procesos comunes a todos los seres humanos de aquellos que varían de una unidad cultural a otra, y el intento por predecir las circunstancias bajo las cuales estas variaciones ocurren.

A continuación se expondrán sucintamente algunos hechos que a mi juicio, son los más destacables en el desarrollo de la Antropología Psicológica. Asimismo, debo recalcar que lo que sigue no pretende ser una expresión acabada de lo que en esencia es esta disciplina, sino más bien entregar antecedentes sobre ella y, advertir a los antropólogos que no sigan descuidando un área que ofrece una gran riqueza científica y que se encuentra, en nuestro país, empíricamente virgen.

\section{La presencia del Psicologismo en el quehacer ANTROPOLÓGICO}

Aunque los estudios antropológicos sobre Cultura y Personalidad se enraízan en el pensamiento e investigaciones prefreudianas, sólo comenzaron a proliferar al darse a conocer las obras de Sigmund Freud.

En los Estados Unidos, los estudios de Antropología Psicológica fueron inspirados por un espíritu crítico hacia la obra de Freud "Tótem y Tabú" (1913). La tesis principal de este libro afirma que tanto el tótem como el tabú surgieron en algún momento de una horda primordial, en la cual el macho más fuerte, celoso y tiránico dominaba a los hombres y expulsaba a los hijos al alcanzar éstos la madurez. En su frustración, los hijos asesinaron al padre, comieron su cuerpo y se apoderaron de las mujeres (madres y hermanas). Luego agobiados por la culpa y el arrepentimiento, renunciaron a las mujeres y se abstuvieron de comer el animal totémico sustituto del padre. Así Freud, sobre la base del mito de la horda primitiva, ofreció una detallada explicación de las reglas totémicas, la exogamia, la prohibición del incesto, la religión y la cultura.

Las ideas vertidas por Freud en "Tótem y Tabú" inspiraron posteriormente una serie de investigaciones dirigidas hacia la corroboración o refutación de las 
mismas. Según Jensen el mencionado estudio suscitó las siguientes contrarrespuestas en la comunidad cientifica:

a) La tesis central de Freud, en la medida en que ella se refiere a la idea de un asesinato real del padre practicado por sus hijos en el tiempo histórico, no puede ser comprobada;

b) las consideraciones de Freud contienen implícitamente la idea de que el supuesto acontecimiento ha tenido efectos duraderos sobre el cerebro y el comportamiento de los hombres a través de un determinado proceso genético hereditario. Investigaciones genéticas posteriores, las cuales no fueron asequibles a Freud, invalidan esa posibilidad. (Dobzhansky);

c) los deseos canibalísticos se dirigen con mayor frecuencia hacia la madre que hacia el padre. El totemismo representaría entonces una defensa cultural ante deseos reprimidos de naturaleza oral y agresiva hacia la madre. (Whiting).

En su época, Freud se enfrentó a duras críticas por parte de los antropólogos. Franz Boaz mantuvo una actitud de profunda hostilidad hacia el psicoanálisis, aunque no dejaba de interesarse por la psicología, su reacción ante el ensayo de Freud fue declarar que el método empleado ni era imparcial ni podía hacer nada por ahondar la comprensión del desarrollo cultural, similar posición sostuvieron Kroeber, (1920-1939) Lowie, y Mead (1930-1963).

Asimismo, su concepto de evolución generó grandes polémicas. Postulaba que la humanidad se había desarrollado igual que un niño que pasa del estado infantil al adulto; según eso, los pueblos ágrafos contemporáneos representaban una etapa detenida de la infancia humana, mientras que el hombre occidental había logrado la madurez mental. Pero, estos primitivos no sólo se asemejaban a los niños, sino que también sus miedos, sus compulsiones, su falta de motivación y la atracción por lo prohibido eran rasgos de individuos neuróticos. De igual manera, su principio de la universalidad del complejo de Edipo tuvo que enfrentar las críticas y la refutación por parte de la antropología, demostrándose empíricamente que el complejo edípico no era universal. Pese a todo, freudianos ortodoxos siguieron sosteniendo la universalidad de este fenómeno psíquico.

En oposición a estas tesis, los antropólogos emprendieron trabajos de campo, tendientes a demostrar que la relación entre cultura y personalidad eran completamente distintas. Se enfatizaba que la cultura modelaba en grado considerable a la personalidad y que, por tanto, había pocos principios universales absolutos. Así, Margared Mead, en su estudio pionero "Coming of age in Samoa" como en el siguiente "Growing up in New Guinea" hizo hincapié en que la naturaleza humana no es rígida e inflexible. Por ejemplo, en su primer estudio, investigó si la rebelión que caracterizaba a la personalidad adolescente en las culturas occidentales era un producto de los cambios biológicos propios de la pubertad, o el resultado de circunstancias culturales. Descubrió que el carácter cultural de los samoanos era en su totalidad, mucho menos emocional que los 
norteamericanos. A los niños de Samoa no se les ocultaba hechos como el nacimiento, la muerte y el sexo; las relaciones sexuales prematrimoniales se consideraban cosa natural y no se exigían fuertes involucraciones emocionales; los adolescentes no se vefan frente a la necesidad de elegir entre una variedad de normas éticas y valores, a menudo antagónicos. O sea, la adolescencia no era un periodo de tormentos ni tensiones, sino simplemente una fase del desarrollo gradual de la vida.

Por su parte, E. Sapir y J. Dollard, a comienzos de la década del 30, dirigieron los primeros seminarios sobre Cultura y Personalidad, en la Universidad de Yale, extendiendo su influencia a muchos otros investigadores. Sapir reconoció a la Psicología como una disciplina susceptible de aplicar a la lingüística, y estudió la manera en que el lenguaje establecia pautas perceptivas y de pensamiento. Las ideas de Sapir fueron desarrolladas por B. Whorf, quien estableció la llamada "Hipótesis Sapi-Whorf ", según la cual existe una relación inextricable entre el lenguaje y la cultura, y las pautas gramaticables de un idioma, a menudo inconscientes, influyen en la manera en que viven y experimentan el mundo quienes lo hablan.

A partir de esos primeros estudios empíricos y de otras demostraciones de la influencia ejercida por la cultura en la formación de la personalidad, varios psicoanalistas especializados comenzaron a analizar culturas ágrafas, manteniendo en varios casos, su ortodoxia freudiana y su convicción sobre la universalidad del simbolismo (R. Roheim, B. Bettelheim). Otros, en cambio, modificaron sus puntos de vista y se inclinaron por temas antropológicos vinculados con la endoculturación y el desarrollo psicológico del niño y la personalidad adulta. Basándose en las afirmaciones freudianas, que la personalidad es desarrollada en la temprana infancia, antropólogos y psicoanalistas comenzaron estudios intensivos priorizando aspectos tales como: el amamantamiento, el destete, el fajamiento, el adiestramiento en materia de higiene personal, la rivalidad entre los hermanos menores, las modalidades de castigo y otras variables condicionantes del carácter (ERICKSON, Gorer, RickMAN).

Así, la mayoría de los psicoanalistas aceptaron el enunciado antropológico de que la cultura es un factor prominente en la formación de la personalidad. Por su parte, algunós seguidores de Boas admitieron que el psicoanálisis presentaba un punto de vista compatible con lo que solía llamarse la "unidad psíquica" de la humanidad. Era innegable que las comparaciones interculturales no serían válidas a menos que se evocaran ciertas constantes psiquicas. Estas actitudes posibilitaron una mayor cooperación entre ambas ciencias.

La cooperación se tradujo en 1937 en la Universidad de Columbia, en seminarios interdisciplinarios, los que consistieron en que algún antropólogo describiese una cultura que había estudiado en el terreno, después de lo cual el neopsicoanalista A. Kardiner hacía una interpretación analítica que luego era discutida en grupo. Los resultados de estos encuentros fueron las obras THE 
INDIVIDU AL AND HIS SOCIETY (1939) The Psychological Frontiers of Society (1945) de Kardiner y The PeOPLe of Alor, de Cora Dubois.

A. Kardiner fue quien realizó una de las contribuciones más importantes al desarrollo del enfoque psicoanalítico dentro de la antropología cultural, al aplicar la técnica del psicoanálisis a los datos empíricos aportados por los antropólogos; estableciendo caracterizaciones psicológicas por medio de los datos culturales. A través del concepto de Personalidad básica (1939) estableció la importancia de ciertos datos de socialización infantil que antes se consideraban secundarios dentro de la antropología cultural. Para él, la "estructura de personalidad básica" consistía en "un conjunto de caracteres psíquicos y de comportamientos derivados de la acción de las instituciones de una sociedad sobre el individuo" (1945, p. 37). Estas instituciones son las disciplinas aplicables a los niños, la instrucción del lenguaje y sus símbolos ideológicos, las técnicas de subsistencia, los sistemas de pensamiento y las constelaciones culturales compartidas por los miembros de una sociedad como resultado de sus primeras experiencias.

Para Kardiner, el proceso de socialización infantil y ciertas instituciones, como la técnica de subsistencia y la organización familiar constituyen las instituciones primarias. En cambio las constelaciones inconscientes que, son la forma en que se expresan las reacciones de personalidad derivadas del proceso de socialización infantil, las llama Instituciones Secundarias o Sistemas Proyectivos, los que tendrían un carácter estable (por ej. el Folklore, Arte y Religión). Estos sistemas proyectivos estarían constituidos por las respuestas de contenido encubiertas, a su vez determinadas por la experiencia del individuo. Como los sistemas proyectivos se incorporan en el individuo, durante el período formativo, y como las experiencias distintas según sea la sociedad, el sistema proyectivo es también distinto.

Plausible es el hecho que Kardiner haya ido más allá de los simples determinismos infantiles, su modelo dinámico permitía explicar el cambio cultural; a saber, si las instituciones primarias cambiaban, producirían modificaciones en la estructura de la personalidad básica, las que a su vez alterarían las instituciones secundarias, por ejemplo, si se cambiara la economía de una sociedad, se alteraría también el sistema de adaptaciones psicológicas de los miembros del grupo social. De esta forma se podría explicar las resistencias al cambio que demuestran los grupos humanos.

Finalmente, sus intentos de "crear una teoría que incorporara hipótesis verificables concernientes a la relación entre la matriz institucional y la personalidad" (HARR1S), no estuvieron exentos de críticas. Las principales refutaciones apuntan a que su teoría sólo puede aplicarse a datos homogéneos y que ésta se ve imposibilitada de explicar cómo se han originado las instituciones primarias.

Muy cercana a los postulados de Kardiner, la notable investigadora Cora Dubois en The PEOPLe Of Alor usó el término Personalidad Modal para explicar 
que la personalidad básica se expresa en el tipo más frecuente de comportamiento individual normativo observado en una sociedad.

La noción de que la cultura generaba una personalidad común se extendió a la idea de que los grupos nacionales poseen "caracteres nacionales". En base a este último enunciado, Ruth Benedict puso en marcha en la Universidad de Columbia, el proyecto de investigación de las culturas contemporáneas. Fue un intento de reconstruir la personalidad cultural desde una perspectiva más ambiciosa y compleja. Benedict, junto a Mead, Metraux, Bateson, Gorer, Goldfrank, Lowie y otros, pretendieron, a través del enfoque conocido como "el estudio de la cultura a distancia" determinar el "carácter nacional" de países modernos.

En su inicio los estudios impulsados por Benedict y sus colaboradores tuvieron una finalidad aplicada, pues persiguieron el objetivo de conocer los modos de conducta característicos de los países aliados y enemigos de los Estados Unidos, en la Segunda Guerra Mundial. Para ello, se colaboró en forma estrecha con sociólogos, psicólogos, economistas e historiadores. Se procedió a analizar filmes, a examinar minuciosamente periódicos, cartas, material de propaganda y otros documentos similares. Asimismo se entrevistó a inmigrantes, solicitándoles historias de vida y aplicándoles test psicológicos. De este modo, metodológicamente hablando, se proporcionó a la ciencia antropológica un nuevo modelo para analizar las regularidades culturales presentes en el carácter de sociedades inaccesibles a la observación directa. En el plano teórico, consideraban que, "los individuos socializados dentro de una misma nación, comparten una tradición específica y poseen ciertas regularidades de conducta culturalmente integradas" (MEAD, 1953, p. 642).

Así planteados, los estudios de carácter nacional se concentraron sobre el modo en que los seres humanos representan a la cultura en la que se han educado. Entre los principales proyectos de relacionar las instituciones de crianza de los niños con la personalidad de los adultos y con las instituciones culturales, destacan las encaminadas a determinar el carácter nacional japonés y ruso. En relación al primer tipo de carácter, G. Gorer expuso la hipótesis de que los japoneses eran un pueblo compulsivo y que su carácter nacional estaba influido principalmente por la severa educación relacionada con el aseo de una edad temprana. Asimismo, este autor atribuyó el carácter nacional de los rusos a las experiencias del fajamiento durante los primeros meses de vida y en los que al niño se lo faja apretadamente con los brazos pegados a los costados (BARNOUw, p. 180).

Los estudios que se derivaron del proyecto de investigaciones contemporáneas fueron ampliamente criticados por los antropólogos, en general, debido a una supuesta debilidad metodológica y al uso de categorías psicoanalíticas para el análisis psicocultural de una moderna nación-estado. Por ejemplo, se sostiene como argumento descalificador que la teoría sobre el carácter japonés fue formulada sin apoyo empírico. Además, más tarde se comprobaría que los 
enunciados vertidos sobre la naturaleza de los hábitos de limpieza japoneses erán equívocos (NORBEck, LANHAM y HARING).

Hacia 1950 había una manifiesta desconfianza sobre la representatividad y confiabilidad científica de los estudios de Cultura y Personalidad. Autores como A. Lindesmith y A.A. Straus expresaban que: "los investigadores en este campo no describen muy claramente ni en detalle cómo han llegado a sus caracterizaciones; la simplificación excesiva y la selección son evidentes y no tienen en cuenta los datos contradictorios; y aunque los resultados no se explican por sí mismos sino que tienen que ser interpretados como los otros datos y, como ellos, pueden estar sujetos a predisposiciones". (Véase BARNOUw, p. 207).

A pesar de lo antojadizo de aquella afirmación, no se puede desconocer lo urgente que se hacía una revisión metodológica y teórica para enfrentar de una forma más efectiva el análisis de los fenómenos psicoculturales.

El estímulo que abrió la puerta a una nueva etapa en la antropología psicológica, lo dio la teoría neoconductista desarrollada por C. Hull. En términos antropológicos, esta teoría significaba que al estudiar el desarrollo de la personalidad debía considerarse toda la gama de interacciones sociales en que se ve envuelto el individuo. Se sumó a este aporte, el uso estadístico del Human Relations Area Files, de G. Murdock. Esta "revolución metodológica", se expresó en el estudio Child Training and Personality, de J. Whitting y J. Child, quienes buscaron correlaciones entre las prácticas de instrucción del nin̄o y las actitudes respecto a las enfermedades en 75 sociedades (DE VOS, p. 14).

Sobre la base expuesta, modificaron y ampliaron la dicotomía primariosecundario de las instituciones de A. Kardiner (1945) proponiendo el siguiente esquema:

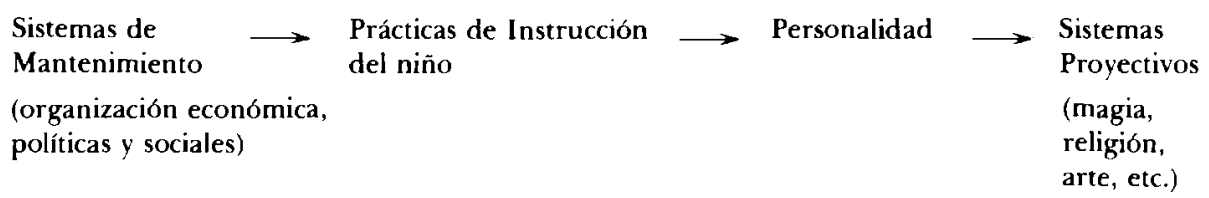

En el citado trabajo, Whitting y Child distinguieron entre los sistemas de conservación de una cultura y sus sistemas de proyección. Se dio por supuesto que la socialización era, en toda cultura, un efecto de los sistemas de conservación y una causa de los sistemas proyectivos. Por ello, este modelo presume que en la medida en que la sociedad afecta a la estructura de hábitos o a la personalidad de los miembros de una sociedad, se convierte en uno de los mecanismos mediante los cuales se integra la cultura.

En su sentido más estricto, "uno de los logros más notables del grupo de Whitting es su demostración de la productividad teórica del concepto de personalidad" (HarRIs, p. 390). Sus estudios ayudaron a vislumbrar la posibilidad de emplear enfoques científicos más rigurosos en la investigación de la personalidad y la cultura. Rompiéndose así, la viciosa circularidad que caracterizaba a los 
estudios tempranos de la antropología psicológica. Hasta entonces se tendía a reconocer la existencia de un núcleo de características de personalidad homogéneas en cada cultura, pero la confrontación de los promedios estadísticos con los rendimientos individuales reales reveló grandes diferencias (Wallace, The Modal personality Structure of the Tuscarora Indians as revealed by the Rorschach test).

Por tanto, la tesis tradicional de la "uniformidad de la personalidad" comenzaba a ser rigurosamente combatida. Es el mismo Wallace (p. 41 ), que comienza a considerar como una alternativa viable la teoría de la organización de la diversidad, según la cual ninguna población, dentro de los límites culturales establecidos puede ser considerada como uniforme con respecto a cualquier variable o norma, no puede afirmarse que hombres y mujeres compartan los mismos valores, los mismos roles cognoscitivos, la misma estructura emocional. Más bien, la cultura constituye un medio de organizar diversas personalidades en vez de producir personalidades homogéneas. Las semejanzas entre las normas de conducta no se consideran resultantes de motivación semejantes, sino que la cultura hace posible la participación individual en actividades de grupo por una diversidad de razones que las motivan; y las interacciones sociales, por ejemplo entre hombres y mujeres, no van a depender de una mutua conformidad con el rol, sino de la complementariedad de los diferentes roles. Para Wallace la participación depende de nuestra capacidad para predecir con alguna certeza la conducta de los demás, y no necesariamente por haber compartido experiencias semejantes durante la niñez.

En definitiva, la novedosa perspectiva de Wallace sugiere que lo que debe ser compartido es el "contrato institucional" que es la cultura. La cual sería el mecanismo que organiza la diversidad individual en matrices sociales viables.

La obra de Wallace, Culture and Personality, no sólo deja entrever el interés de la antropología por comprender la relación entre la cultura y las estructuras mentales y los procesos de los individuos - debido principalmente a los estudios de cognición en la psicología cognitiva - sino que, además, se le puede considerar pionera en la aplicación del "enfoque orgánico". (Ver cap. V).

Ante las nuevas y continuas evidencias se hacía difícil mantener la suposición de la facilidad de establecer la personalidad básica o modal. Esto no significaba desligitimar totalmente la influencia de la socialización temprana en la conformación de la personalidad del individuo, sino más bien, esa influencia iba más allá de las pautas más o menos concientes de amamantamiento o destete. Se debía buscar, tambien, otros aspectos que afecten la conducta y la personalidad. Llegándose a considerar, por ejemplo, la relación del organismo con el medio (Clima, dieta, nutrición, etc.) (Whiting, Kıuckhon, Anthony, Whiting).

En la actualidad, los antropólogos reconocen que los individuos aprenden ciertos modelos de comportamiento, pero no aceptan la idea de que ni siquiera la estructura profunda de las personalidades es standard. Pues, los procesos biológicos parecen ejercer una influencia importante sobre la formación de la personalidad (KLuCKhon, MurRay, pp. $71-85$ ). En segundo lugar, los individuos 
están expuestos a diferentes situaciones vitales y, en consecuencia, internalizan aspectos diversos de la cultura. Además, la existencia de una variedad de estructuras cognoscitivas y motivacionales no es incompatible con un conjunto de expectativas o interacciones mutuas y organizadas, indispensable para la vida social.

En el presente, es indiscutible el hecho que la antropología psicológica se erige como una área de estudio fértil y significativa para el desarrollo de la ciencia antropológica. Esta apología se sustenta en una de las más relevantes peculiaridades que exhibe este campo de estudio: la amplia variedad de temas a los que presta atención y al número de disciplinas a las que acude para estudiarlos.

Lejanos están esos días en que la tradición durckeniana envolvía el pensamiento de los sociólogos y antropólogos con sus aseveraciones de que los "hechos sociales" deben ser explicados con independencia de los fenómenos biológicos, psicológicos o de cualquier otra ciencia. A pesar de que esa tradición levantó, sobre todo, en la antropología social inglesa un gran escudo para protegerse de la incorporación de la dimensión psicológica a sus estudios, muchos de sus exponentes no estuvieron ajenos al "pecado" de recurrir a conceptos psicologistas, cognitivos y emocionales en variados análisis de situaciones "estrictamente sociales" (R. FIRTH, p. 124), (M. ForTes, p. 42).

No obstante, que se niegue, el "psicologismo es un hábito arraigado entre los antropólogos sociales" (HARRIS, p. 343). Nada más revelador en esta discusión es la siguiente frase de M. Mead: "La tendencia a ver en algunos trabajos antropologicos problemas de la 'cultura y la personalidad', y a negar su existencia en otros, es una realidad, una tentativa de trazar una artificial línea divisoria en determinado punto de la formulación del problema. En realidad se trata de una línea continua, en uno de cuyos extremos el trabajo etnográfico formal (lo mismo que la mayor parte de la teoría social) esquiva la cuestión con afirmaciones "él era mucho más frecuente entre las tribus de la costa" o "durante el siglo siguiente, la situación se tornó mucho más inestable, y eran frecuentes el robo y el asesinato" sin plantear problema de los correlativos de la cambiante incidencia del fenómeno de la violencia, existente en la organización intrasíquica de los individuos" (pp. 121-122).

La Antropologia Psicologica de hoy aboga por estudios interdisciplinarios y la difusión de conceptos operativos en miras a una mayor solidez de los análisis socio-culturales. Por ello intenta suprimir la dicotomía representada por lo etnológico y lo psicológico, reduciendo las significaciones independientes. Lo que se intenta es explicar lo etnológico y lo psicológico como conceptos de una misma realidad empírica; trascendiendo las diferencias que impone la metodología propia de cada disciplina y, así, lograr incorporar dos técnicas a un método analítico común.

De igual manera, la Antropología Psicológica ha tendido a reducir las distancias con otras disciplinas cientificas, asi, se nutre continuamente de los 
aportes de las psicologías, la psiquiatría, la neurología, la etología, etc. Configurándose como disciplina interdisciplinaria.

A manera de síntesis, Esteva (p. 40) considera que la Antropología Psicologica es un campo de la antropología cultural que combina los datos psicológicos -proyectivos, profundos y de comportamientos visualizados- observados en los individuos de una sociedad en términos de sus categorías fundamentales de expresión: 1) forma material del medio y de sus instrumentos de acción (tecnología) para las funciones económicas; 2) organización y estructura social de la producción material y distribución de sus miembros según el sistema regulador de rol status; y, 3) ideología, racionalizaciones y eidos de los sistemas axiológicos que guían la acción social. Estas tres variables homogeneizadas en un modelo etnográfico constituyen el fundamento sobre el que descansan los análisis de la antropología psicológica.

Es indudable que esta disciplina en los últimos años se ha desarrollado aceleradamente, también es cierto que esa consolidación científica se ha dado preferentemente en Estados Unidos y que los investigadores latinoamericanos han estado ausentes de obra en ese crecimiento. Por ende, el campo de la antropología psicológica en nuestros países permanece casi intacto empíricamente. Es más, los marcos analíticos empleados resultan ser préstamos que generalmente son producto de experiencias en realidades culturales distintas a las nuestras. Por tanto, necesitamos imperiosamente desarrollar teorías relativas al ámbito de nuestras realidades históricas. Para este fin, los Departamentos de Antropología latinoamericanos juegan un rol decidor, y deben incentivar la investigación profunda de los sistemas socio-culturales por medio de nexos interdisciplinarios y, así, paulatinamente, romper la dependencia intelectual respecto a categorías de análisis que si bien son relevantes para ciertos contextos no necesariamente son factibles de usar para lograr comprender nuestra dinámica social.

No puedo concluir, sin expresar que la interdisciplinariedad que enmarca a los estudios de la disciplina que hemos venido tratando debería, también, guiar el trabajo de todos los antropólogos, éstos no pueden desconocer el hecho de que si desean ser certeros en sus postulados deben extender su preparación y sus intereses hacia otros campos e incorporar a su propio pensamiento los datos y conceptos de otras disciplinas.

\section{Bibliografía}

Arnold, Marcelo, Cultura y Personalidad, 1980 (mimeog.).

Barnouw, Victor, Cultura y Personalidad, Buenos Aires, Edit. Troquel, Biblioteca El tema del hombre, 1967.

Benedict, Reth, El hombre y la cultura, Buenos Aires, Edit. Sudamericana, 1939.

Bock, PhILIP, Continuities in Psychological Anthropology, W.H. Freman and Company, 1980.

De Vos, George, Antropología Psicológica, México, Edit. Anagrama, 1980. 
De WaAl, A. Marie, Imágenes del hombre. Buenos Aires, Amorrortu, 1983.

Dobzhansky, T., Mankind evolving: The evolution of the human species, London, New Haven, 1962.

Dubois, Cora, The people of Alor. Minneapolis, Univ. of Minnesota, 1944.

Esteva, Clavdo, Cullura, Sociedad y Personalidad, Barcelona, Edit. Promoción cultural. 1978.

FiRth, Raymond, Essays on social organization and values, London, Althtone, 1963.

Freld, Sigmund, Totem y Tabú, Buenos Aires, Edit. Troquel, 1966.

Goldfrank, Esther, "Socialization, Personality and the Structure of Pueblo Society", American Anthropologisl, vol. xLv11, 1945, pp. 516-539.

Haefner, Carlos, La aculluración en migranles forzados: un estudio exploralorio descriptivo, Tesis para optar al Grado de Licenciado en Antropología, Departamento de Antropología, Facultad de Filosofía, Humanidades y Educación, Universidad de Chile, Santiago, 1984

Hakr1s, M., The rise of Anthropology theory, Edit. Thomas Crowell Company, 1969.

Hst. F., Psychological Anthropology: approaches to cullure and personality, Dorsey Press Inc., 1961.

HLNt, E., Personalities and cultures: readings Psychological Anthropology, Texas Press, 1976.

Jensen, H., "Psicoanálisis y Antropología", Revista de Ciencias Sociales [San José], Ed. especial $N^{\circ} 4$, Universidad de Costa Rica, 1984.

Johnson, A., Cuantifications in Cultural Anthropology, Univ. Yale, 1978.

Kardiner, A., El Individuo y su sociedad. México, Fondo de Cultura Económica, 1945.

KARDiner, A., Las fronteras psicológicas de la sociedad. México, Fondo de Cultura Económica, 1955.

Klineberg, O., Psicología Social, México, Fondo de Cultura Económica, 1963.

Kllckhohn, C. y Murray, H., La personalidad en la naturaleza, la sociedad, la cultura, Buenos Aires, Edit. Grijaldo, 1969.

Kroeber, A., "Totem and Taboo. An Ethnologic Psychoanalysis", American Anthropologist, vol. 22, 1920, pp. 40-55.

Linton, R., Cultura y Personalidad, México, Fondo de Cultura Económica, 1945.

Lowie, R., The german people, New York, Farrar and Rinehart, 1945.

MaLinowsky, B., Sex and represion in savage society, New York, Meridian Books, 1927.

Malinowsky, B., Una teoría científica de la cultura, Buenos Aires, Edit. Sudamericana, 1948.

Malinowsky, B., Estudios de psicología primitiva, Buenos Aires, Edit. Paidós, 1963.

MEAD, M., Adolescencia y cultura en Samoa, Buenos Aires, Edit. Abril, 1945.

MEAD, M., Sexo y temperamento, Buenos Aires, Edit. Paidós, 1963

Mead, M. et al, Cultura y sociedad, Buenos Aires, Edit. Libros Básicos, 1965.

Mercier, R., Historia de la Antropologia, Edit. Península, 1969.

Wallace, A., Culture and personality, New York, Random House, 1961.

Whiting, J. y Child, I., Child training and personality, H. Hover, Yale University Press, 1953. 